\title{
Les chemins de l'histoire du pouvoir dans le médiévisme portugais (ca 1970-ca 2000)
}

Judite A. Gonçalves de Freitas

\section{(2) OpenEdition}

1 Journals

Édition électronique

URL : https://journals.openedition.org/cem/927

DOI : $10.4000 /$ cem. 927

ISSN : 1954-3093

Éditeur

Centre d'études médiévales Saint-Germain d'Auxerre

Édition imprimée

Date de publication : 15 août 2004

ISSN : 1623-5770

Référence électronique

Judite A. Gonçalves de Freitas, « Les chemins de l'histoire du pouvoir dans le médiévisme portugais (ca 1970-ca 2000) », Bulletin du centre d'études médiévales d'Auxerre / BUCEMA [En ligne], 8 | 2004, mis en ligne le 20 décembre 2006, consulté le 22 septembre 2022. URL : http://journals.openedition.org/ cem/927 ; DOI : https://doi.org/10.4000/cem.927

Ce document a été généré automatiquement le 22 septembre 2022.

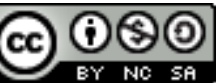

Creative Commons - Attribution - Pas d'Utilisation Commerciale - Partage dans les Mêmes Conditions 4.0 International - CC BY-NC-SA 4.0

https://creativecommons.org/licenses/by-nc-sa/4.0/ 


\title{
Les chemins de l'histoire du pouvoir dans le médiévisme portugais (ca 1970-ca 2000)
}

\author{
Judite A. Gonçalves de Freitas
}

$1 \quad$ Y a-t-il une nouvelle histoire politique du Moyen Âge portugais ? Ce travail prétend répondre à cette question, en suivant les divers chemins du politique dans l'historiographie médiéviste, en les articulant avec l'institution, par excellence créatrice des savoirs : l'université. En partant de la présentation des principales lignes de recherche, pratiquées ou en cours, et des orientations intellectuelles, nous ferons un bilan de la plus récente historiographie du politique concernant la fin du Moyen Âge au Portugal.

2 À première vue, la carte actuelle de l'historiographie portugaise met en relief l'inexistence d'une distribution temporelle équilibrée des recherches. Cette distribution est justifiable par la valorisation naturelle, de la part de la communauté des historiens, des thématiques centrées sur les aspects de l'organisation et de l'affirmation politique du Portugal du Moyen Âge dans le contexte péninsulaire. Ainsi, on comprend aisément la plus grande incidence de la pratique historiographique dans les périodes correspondant au Moyen Âge central (XI ${ }^{\mathrm{e}}$-XIII ${ }^{\mathrm{e}}$ siècle) et au Moyen Âge tardif (XIV ${ }^{\mathrm{e}} \mathrm{XV}^{\mathrm{e}}$ siècle). Le premier Moyen Âge ( $\mathrm{V}^{\mathrm{e}}-\mathrm{X}^{\mathrm{e}}$ siècle) a toujours été, par tradition, un temps de plus grand voisinage avec l'Antiquité tardive, et correspond à la période qui précède la formation du Portugal en tant qu'unité politique indépendante (royaume) ${ }^{1}$. Au Portugal, les plus grands universitaires spécialistes de cette période proviennent de la philologie, de l'histoire de la philosophie et quelques-uns de l'histoire de l'art.

3 La recherche se centre sur la re-émergence du politique dans le médiévisme tardif portugais (ca 1970-ca 2000). Nous poursuivrons avec la référence aux thématiques effectuées par les spécialistes de la problématique des pouvoirs, et considérerons surtout ceux qui les représentent et qui se sont engagés à les développer. De la même façon, nous attirerons l'attention sur les lacunes existantes en tenant compte des chemins actuels parcourus par le politique ${ }^{2}$. 
4 Nous articulerons cette réflexion autour de cinq axes d'investigation qui caractérisent la plus récente historiographie du politique dans le Moyen Âge tardif portugais, à savoir : les pouvoirs (pluralité et inter-relations), le pouvoir royal (institutions et société politique), les pouvoirs locaux (institutionnalisation et “élitisation"), le pouvoir seigneurial laïque et les pouvoirs ecclésiastiques.

La re-émergence du politique dans le médiévisme tardif portugais (ca 1970- ca 2000)

5 Dans la ligne récente de l'historiographie du politique des vingt-cinq dernières années, les centres d'intérêt des historiens présentent une plus grande diversité dans la définition des lignes d'investigation et dans l'enrichissement des problématiques étudiées.

6 L'analyse des facteurs sociaux et politiques responsables des conflits des noyaux liés aux sphères du pouvoir est présente dans la recherche d'Humberto B. Moreno (1973). Ainsi, l'auteur procède à la justification du conflit d'Alfarrobeira (1449) à travers l'élaboration systématique de 216 micro-biographies de partisans du roi Alphonse $\mathrm{V}$ et de l'Infant Pierre ${ }^{3}$. La thèse de doctorat de Luís A. Fonseca (1975), sur la figure du Connétable Pierre, fils de l'ex-régent de même nom (1439-1448), se situe dans la même orientation et perspective méthodologique ${ }^{4}$. Quant à António M. Hespanha, il rapproche l'histoire du pouvoir avec l'histoire du droit ${ }^{5}$. L'auteur part d'une réflexion qui vise à connaître l'efficacité de l'appareil administratif de la Couronne à travers l'analyse de l'amplitude du pouvoir de la royauté et des limites imposées par les autres pouvoirs, principalement pour les siècles de "l'Ancien Régime" ${ }^{6}$. De la même manière, il conduit une réflexion méthodologique sur l'emploi des sources (analyse et critique) ${ }^{7}$.

7 Mais c'est sous l'influence de l'historiographie étrangère, surtout française ${ }^{8}$, que de nouvelles investigations de l'histoire politique médiévale portugaise apparaissent. Dans les années quatre-vingt, une nouvelle génération d'historiens du politique s'affirme par l'étude des diverses micro-populations (serveurs royaux, élites municipales, aristocratie laïque...) en recourant à de nouveaux supports méthodologiques comme la prosopographie 9 .

8 L'histoire sociale des pouvoirs a été explicitement reconnue comme un lien profitable vers d'autres sciences humaines - la sociologie, l'anthropologie, la science politique ou la statistique appliquée. En simultané, on a aussi opéré une utilisation novatrice des ressources de la diplomatique pour aborder les sources indispensables à l'étude de la problématique des "pouvoirs".

Thèmes et problèmesLes pouvoirs : pluralité et inter-relations

9 Actuellement, il n'est pas abusif d'observer que l'État moderne, envisagé comme une forme d'organisation politique en formation depuis la fin $\mathrm{du} \mathrm{XIII}^{\mathrm{e}}$ siècle dans presque tous les territoires de l'Occident médiéval, est inévitablement lié à la définition et aux consolidations de l'identité/unité des autres structures du pouvoir, c'est-à-dire le pouvoir des villes et des élites locales, le pouvoir seigneurial laïque ou les pouvoirs ecclésiastiques. L'État moderne surgit à partir du moment où il commence à se réserver les domaines $d u$ politique et $d u$ "publique", les distinguant d'abord $d u$ domaine spirituel et ensuite du domaine privé ${ }^{10}$. L'autorité souveraine se manifeste par l'exercice effectif des pouvoirs et des moyens conçus par le monarque pour arriver à l'imposer.

Les analyses historiographiques plus récentes sont tournées vers la connaissance de ces formes d'organisation et l'institutionnalisation des multiples pouvoirs. Elles définissent les profils structuraux, les spécificités, les limites, les comportements et 
les stratégies et, de façon complémentaire, les agents du pouvoir et l'encadrement spatio-temporel de son exercice ${ }^{11}$.

11 La hiérarchie des anneaux politiques établis par la monarchie à la fin du Moyen Âge portugais montre une nécessaire mise en perspective des relations du pouvoir royal avec les autres détenteurs de pouvoirs, et ceci malgré les "vides" territoriaux ${ }^{12}$ et/ou les cercles juridictionnels ${ }^{13}$ qui interceptent les rayons d'action des structures gouvernementales centrales et régionales du souverain ${ }^{14}$.

L'affirmation du pouvoir politique est aussi déterminée par les modèles de rapports du monarque avec la noblesse. En Hispanie, aux XIV et $\mathrm{XV}^{\mathrm{e}}$ siècles, la noblesse, malgré les transformations constatées, garde le prestige, le respect et les faveurs de l'autorité royale ${ }^{15}$. En vérité, on assiste à "une progressive prédominance de la noblesse de cour (à partir $\mathrm{du} \mathrm{XIV}^{\mathrm{e}}$ siècle) sur la noblesse seigneuriale et par conséquent une croissante dépendance de la noblesse face au pouvoir publique" ${ }^{16}$. Le monarque continue à faire surtout confiance aux nobles qui demeurent à la cour et qu'il considère comme ses serviteurs. La noblesse reste un soutien indispensable au monarque.

Les rapports du monarque avec l'Église et les seigneurs ecclésiastiques paraissent plus problématiques. Les confrontations dans ce domaine semblent avoir été "très fréquentes" et remontent, selon une étude récente, au XIII ${ }^{\mathrm{e}}$ siècle ${ }^{17}$.

14 Les rapports entre les différentes cellules de pouvoir à la fin du Moyen Âge portugais ont occupé, dans les deux dernières décennies du $\mathrm{XX}^{\mathrm{e}}$ siècle, de nombreux médiévistes. Ils ont essayé de comprendre la dynamique de l'exercice de la souveraineté royale dans le faisceau complexe des pouvoirs déjà cités.

Le pouvoir royal : institutions et société politique

15 Sans oublier les considérations établies sur l'historiographie portugaise récente, il est impérieux d'observer que la réhabilitation de l'histoire politique en tant qu'histoire des pouvoirs ou du pouvoir a été profondément liée. et l'est encore. à l'activité de recherche d'Armando Luís de Carvalho Homem, de l'université de Porto ${ }^{18}$. Cet universitaire a fait de l'histoire politique un objet légitime de la curiosité des historiens, lui conférant de l'autonomie en tant que champ de recherche. Il a été aussi le précurseur et l'instigateur de certaines des méthodes d'investigation de la matière. Dans un premier travail, il a étudié les Aspectos da administração portuguesa no reinado de D. Pedro $I^{19}$, puis, dans sa thèse de doctorat soutenue en $1985{ }^{20}$, il procède au traitement exhaustif des actes royaux relatifs à la grâce, à la justice, aux finances et à l'administration générale, en établissant et en défendant une typologie systématique des diplômes royaux. Recourant à la méthode prosoprographique ${ }^{21}$, il dessine le profil biographique de l'officialité, recherchant une interprétation sociologique du fonctionnement des organes du pouvoir royal, en incluant le Conseil ${ }^{22}$. En 1990, il fait un premier bilan des réflexions méthodologiques qu'il a produites sur l'État et son organisation ${ }^{23}$.

16 Sous son orientation scientifique, dans le domaine de l'histoire de l'État et de ses serviteurs ${ }^{24}$, a été développé l'essentiel des recherches sur l'administration royale et le pouvoir central dans les règnes des rois Eduardo, Alphonse V et Jean II ${ }^{25}$. En 1989, Eugénia P. Mota défend un mémoire de maîtrise (Do "Africano" ao "Príncipe Perfeito" (1480-1483). Caminhos da burocracia régia) qui traite de questions méthodologiques sur l'apport de la prosopographie dans le principal milieu de l'officialité royale, c'est-à-dire celui des officiers rédacteurs des lettres. En 1991, je réalise une étude semblable sur les deux niveaux de serviteurs (rédacteurs et scribes) dans le règne de "l'Éloquent", et élabore les notices prosopographiques de 139 individus ${ }^{26}$. Huit ans plus tard, dans ma 
dissertation de doctorat, en recourant aux mêmes méthodes de recherche et de traitement des sources, je procède à un approfondissement de l'analyse du fonctionnement de l'appareil d'État et à la caractérisation "globale" de la société politique du règne d'Alphonse $\mathrm{V}$ (1439-1460) en cataloguant 415 nouveaux officiers ${ }^{27}$.

Désormais, de nombreuses monographies sont présentées sur le pouvoir central et les principaux noyaux d'officiers de la bureaucratie royale (rédacteurs et scribes) ${ }^{28}$, et, depuis les années quatre-vingt-quinze, un grand intérêt se manifeste pour la connaissance de la durée de vie et des carrières des individus des groupes analysés ${ }^{29}$. Tel que nous l'avons déjà dit, “(...) pour l'historien des sociétés politiques, [s'est imposé] le besoin impérieux d'augmenter le champ de ses recherches aux multiples dimensions de la connaissance sociale ${ }^{30}$ ".

18 L'historiographie récente, sur l'administration monarchique et les serviteurs royaux, fournit un état assez complet des chemins de la bureaucratie royale entre 1310 et 1483 et, mutatis mutandis, de la typologie des diplômes enregistrés par la chancellerie. Plus récemment, à propos du cadre de la production législative, les principales questions, depuis la fin du XIII ${ }^{e}$ jusqu'au début du XVI ${ }^{\mathrm{e}}$ siècle, ont été résolues par Armando L. de C. Homem ${ }^{31}$. Pour connaître le modèle bureaucratique de l'administration de la justice à la fin du Moyen Âge portugais nous devrons recourir à la dissertation de doctorat de Luís M. Duarte, qui traite spécifiquement les diplômes de "pardon" issus de la chancellerie alphonsine, et propose ainsi une lecture de caractère sociologique de la criminalité dans les deux dernières décennies du règne en question ${ }^{32}$.

Le Portugal du Moyen Âge tardif a également développé certaines modalités de l'utilisation de la force des armes, quoique le pouvoir central ne détienne pas encore "le monopole de l'exercice de la violence". João G. Monteiro, de l'université de Coïmbre, le plus grand spécialiste en histoire sociale de la guerre, a entrepris des études dans ce domaine ${ }^{33}$, avec comme références les travaux, entre autres, de Peter E. Russel, Philippe Contamine et Christopher Allmand ${ }^{34}$.

Quant à la structure de l'administration financière ou de la fiscalité publique ${ }^{35}$, il faut mentionner la précoce et innovatrice réflexion de Vitorino M. Godinho publiée en 1968 : "Finanças Públicas e estruturas do Estado". C'est Iria Gonçalves qui a traité quelques aspects des finances royales du bas Moyen Âge ${ }^{36}$, notamment ceux qui se rapportent aux droits royaux et aux principaux instruments d'imposition (de tribut) appliqués par les monarques ${ }^{37}$.

21 Cependant, nous ne disposons pas actuellement d'une étude de fond sur cette importante dimension de la "genèse de l'État moderne", et ceci se doit, peut-être, à l'absence de programmes systématiques de recherche dans les universités portugaises.

Dans l'historiographie des cours, assemblées politiques (cortes) en tant qu'espaces de représentation et de résistance, il faut consulter les travaux d'Armindo de Sousa qui, depuis 1987, a apporté une précieuse contribution à l'approfondissement de la connaissance de ces assemblées, en disséquant de façon critique les chapitres généraux municipaux ${ }^{38}$. Les problématiques de cet auteur sont proches de l'histoire des mentalités politiques ${ }^{39}$.

Dans une ligne de recherche adjacente, que nous pourrions désigner par histoire des cultures politiques, se distingue le travail de Rita C. Gomes sur "La Cour des rois du Portugal à la fin du Moyen Âge" ${ }^{40}$. Prenant pour objet d'étude la cour, elle se jette dans l'histoire des structures de parenté des familles des officiers dans un mélange 
d'influences d'anthropologie historique à la française et d'historiographies allemande, italienne ou anglo-saxonne ${ }^{41}$. Dans le domaine de la représentation ou du cérémonial de la royauté s'inscrit la réflexion sur les lieux du pouvoir, avec des contributions venues de l'histoire, de la sociologie, de l'histoire de l'art, de la géographie et de l'architecture ${ }^{42}$.

Le panorama de l'édition de sources s'est considérablement amélioré depuis le début des années quatre-vingt ${ }^{43}$. António H. de O. Marques et João A. Dias, de l'université Nova de Lisbonne, en sont les principaux responsables. Les registres de la chancellerie royale et les chapitres des cours sont les secteurs de publication privilégiés ${ }^{44}$.

Les pouvoirs locaux : institutionnalisation et "élitisation"

La "nouvelle histoire politique" s'est également intéressée à l'articulation du pouvoir royal et du pouvoir municipal ${ }^{45}$, à l'histoire de l'administration municipale ${ }^{46}$ et, finalement, à l'étude de l'exercice du pouvoir par les élites municipales ${ }^{47}$.

C'est José Mattoso qui a jeté un regard neuf sur la problématique des municipalités ${ }^{48}$. En 1986, Maria Helena da C. Coelho et Joaquim R. Magalhães, de l'université de Coïmbre, ont approfondi et délimité d'un point de vue conceptuel ou "instrumental", les racines du pouvoir municipal du bas Moyen Âge à l'assemblée constituante (1821-1822) ${ }^{49}$.

Depuis le début des années quatre-vingt, dans l'étude des pouvoirs municipaux, se détachent les travaux de Maria Helena da C. Coelho, regroupés dans les années quatrevingt-dix dans la publication Homens, Espaços e Poderes. Séculos XI-XVI ${ }^{50}$. D'autres travaux sont à signaler, en particulier des doctorats, sur l'Entre-Minho-e-Lima, Évora, Guimarães, Torres Vedras et óbidos ${ }^{51}$.

L'étude de l'histoire des sociétés urbaines a aussi compté sur la contribution de António H. de O. Marques et d'Iria Gonçalves ${ }^{52}$. Entre 1985 et 1999, ils ont élaboré, par la rédaction de monographies, une vaste enquête sur des villes, notamment Abrantes, Alenquer, Aveiro, Cascais, Chaves, Elvas, Guimarães, Porto de Mós, Setúbal, Silves, Tomar et Torres Vedras. La majorité de ces études débute dans la période antérieure à la genèse de l'État moderne, car il y a des décalages chronologiques dans le système de relations avec le pouvoir central.

Dans le cadre institutionnel de l'exercice du pouvoir par les élites urbaines, d'autres études ont été élaborées, recourant ponctuellement à la prosopographie, sur les élites de Porto (Adelaide M. da Costa ${ }^{53}$ ) et de Funchal (Miguel J. Rodrigues ${ }^{54}$ ). C'est sous le patronage d'Humberto B. Moreno que l'on développe à l'université de Porto la connaissance de l'édilité de cette ville grâce à des mémoires de maîtrise. En ce qui concerne les sources du pouvoir municipal, il y a un retard significatif comparativement aux sources du pouvoir central, notamment pour les actes des conseils ${ }^{55}$ et d'autres documents municipaux ${ }^{56}$.

Le pouvoir seigneurial laïque

30 Les premières contributions de fond qui ont fait école sont celles de José Mattoso ${ }^{57}$. Cet auteur, actuellement retraité, a suscité l'élaboration de recherches sous la forme de monographies sur l'histoire sociale de la noblesse, par le biais de la reconstitution systématique de généalogies, d'études de tombeaux, de résidences seigneuriales, de définitions des bases territoriales et régionales des fortunes et comportements de lignage. Parmi les universitaires qui abordent ces sujets, il faut détacher : Luís Krus ${ }^{58}$ et António R. de Oliveira ${ }^{59}$ qui traitent de la culture de la noblesse ${ }^{60}$; Leontina Ventura qui se 
penche sur la noblesse de la cour de Deux cent et sur les bases régionales du pouvoir de la noblesse de Coïmbre ${ }^{61}$; Bernardo V. e Sousa dont l'étude porte sur les stratégies de sauvegarde du succès social d'une famille noble (les Pimentéis) ${ }^{62} ;$ José A. Pizarro qui reconstitue les généalogies de familles de premier, deuxième et troisième plans dans le règne du roi Denis ${ }^{63}$; et enfin Rita $\mathrm{C}$. Gomes qui travaille sur la société de la cour à la fin du Moyen Âge.

31 En ce qui concerne l'étude systématique du mouvement de constitution des maisons seigneuriales soustraites à la juridiction royale au $\mathrm{XV}^{\mathrm{e}}$ siècle ${ }^{64}$, on peut signaler les contributions de João S. de Sousa sur la Maison de l'Infant Enrique ${ }^{65}$, de Mafalda S. da Cunha sur la Maison de Bragança ${ }^{66}$ et de Luís F. Oliveira sur la Maison des Coutinhos ${ }^{67}$. Maria de L. Rosa préfère suivre les thématiques développées par Jean-Claude Schmitt et propose une étude empirique des origines et de l'évolution du droit d'aînesse aux XIV $\mathrm{XV}^{\mathrm{e}}$ siècles ${ }^{68}$, et démontre ainsi l'influence récente parmi nous de l'anthropologie historique française ${ }^{69}$.

En somme, dans le domaine des études de la noblesse du bas Moyen Âge, nous assistons à un renouvellement méthodologique des problématiques, dont les exemples sont l'introduction à l'histoire des images du pouvoir, à l'histoire de la représentation et à l'histoire de la parenté.

Les pouvoirs ecclésiastiques

Dans ce que nous pouvons actuellement considérer le "classique" engagement entre le pouvoir royal et les pouvoirs ecclésiastiques, il faut mentionner José Marques et sa recherche pionnière sur l'archevêché de Braga au $\mathrm{XV}^{\mathrm{e}}$ siècle, travail exemplaire qui a privilégié les implications du pouvoir ecclésiastique avec le pouvoir monarchique ${ }^{70}$. Récemment, il a fait le bilan de l'“espace" relationnel entre les deux pouvoirs tout au long du Moyen Âge portugais ${ }^{71}$. Les travaux de Margarida G. Ventura suivent la même voie car elle étudie les formes d'action du pouvoir royal en conformité avec les résistances du pouvoir du clergé, cherchant à dévoiler la capacité et les abus de chacun 72 .

Dans cette thématique des rapports entre pouvoirs, nous devons encore mettre en relief le travail d'Hermínia V. Vilar sur le diocèse d'Évora, qui a envisagé une structure de pouvoir et un instrument de domination ${ }^{73}$. À la suite des recherches actuelles sur la structure des pouvoirs ecclésiastiques, des monographies sur les diocèses de Coïmbre et de Lamego sont en préparation. Les manques concernent spécifiquement les diocèses de Porto et de Viseu. Depuis la fin des années quatre-vingtdix, l'université de Minho, a mis en place un projet aux problématiques semblables, qui vise à étudier de façon systématique les archives de l'archevêché de Braga ${ }^{74}$. On attend que ce plan de recherche fournisse une vision précise des détenteurs de charges et de la hiérarchie des attributions du personnel ecclésiastique du diocèse de Braga.

Conclusions

Nous avons essayé de faire une synthèse des principaux axes de recherche, pratiqués ou en cours, les articulant avec les effets de l'internationalisation des problématiques.

Sur un plan global, nous pouvons dire que trois lignes de force découlent des changements épistémologiques de la nouvelle histoire politique : la première s'attache au changement des modèles historiographiques de la recherche ; la deuxième passe par la plus grande diversité thématique ; et la troisième se trouve liée à l'intégration 
transdisciplinaire de l'histoire avec les autres sciences humaines, notamment la sociologie et l'anthropologie ${ }^{75}$.

En ce qui concerne les domaines de l'histoire des pouvoirs, nous sommes en mesure d'affirmer que la fragmentation de la recherche, qui n'est pas excessive au Portugal, a permis l'approfondissement thématique et a ouvert les portes, d'une certaine façon, a l'ajustement des problématiques ${ }^{76}$.

38 Nous pouvons conclure, si nous tenons compte de ce qui a été dit, qu'il y a des orientations d'investigation très diverses, empreintes, non seulement de la "culture" des lieux de production des savoirs historiographiques. universités -, mais aussi du choix, de la part de certains universitaires, d'un discours personnel "teinté" des courants post-modernes qui valorisent "la toujours éminente irruption du nouveau", sans qu'il y ait toujours un accueil critique ${ }^{77}$. Ainsi, nous avons assisté très récemment à l'augmentation du nombre des études développées sur une micro-échelle ${ }^{78}$, même si l'histoire des pouvoirs n'est pas celle qui est le plus affectée par ce courant ${ }^{79}$.

39 En tenant compte de la sélection des thèmes de l'historiographie la plus récente, il y a, fondamentalement, deux lignes d'action dans la recherche de la nouvelle histoire du politique du bas Moyen Âge portugais :

40 - une histoire sociale et institutionnelle des pouvoirs, qui tend, par la main de certains de ses représentants, vers une histoire des mentalités politiques ;

41 - une histoire des représentations et images du pouvoir, avec des variantes pour l'histoire des comportements et des structures de parenté, très sensible aux interprétations sociologiques et anthropologiques originaires de l'École allemande ou de l'historiographie française, qui a le sceau de l'École des hautes études en sciences sociales (EHESS) ${ }^{80}$.

42 Malgré les lacunes et les infléchissements signalés, nous ne pouvons pas négliger l'importance des notes de complémentarité entre la plupart des thématiques développées, quoiqu'elles cohabitent, dans la recherche, avec des projets individuels et plus singuliers.

43 En conclusion, au Portugal dans un avenir proche, d'autres "carrefours" se devinent sur les chemins de l'histoire des pouvoirs. Espérons que ses meilleurs exemples pourront conduire à l'édification d'un tout rationnel et cohérent.

\section{NOTES}

1.L'archéologie, dans une première phase, depuis les années soixante-dix, est apparue dans les cours d'histoire, coïncidant, d'une certaine façon, dans les curricula avec des thématiques du premier Moyen Âge. Ce n'est qu'entre 1978 et 1981 qu'elle apparaît comme une variante avec l'histoire de l'art, puis en 1981 comme variante proprement dite et en 1999 comme grade de licence. Toutefois, en tant que spécialité de doctorat, elle apparaît depuis les années soixante-dix. Les premiers maîtres apparaissent dans les années quatre-vingt. 
2.Voir une excellente vision des principales incidences thématiques du médiévisme portugais après avril 1974 dans A. L. de C. HOMEM, “O Medievismo em liberdade (anos 70/anos 90)", dans M. C. PROENÇA, sous la dir., Um século de ensino da História, Lisbonne, 2001, p. 183-216.

3.A Batalha de Alfarrobeira : Antecedentes e significado histórico, 2 vol., Coïmbre, 1979 (rééd.).

4.0 Condestável D. Pedro de Portugal, Porto, 1982.

5.Sur les traditions de cette histoire, voir un bilan récent dans Cultura Jurídica Europeia Síntese de um Milénio, Mem Martins, 2003.

6.História das Instituições. Épocas Medieval e Moderna, Coïmbre, 1992 (rééd.) ; Id., As Vésperas do Leviathan. Instituições e poder político. Portugal, século XVII, 2 vol., Lisbonne, 1987. Pour la nouvelle conception du politique, même pour les temps modernes, voir A. M. HESPANHA, “O Antigo Regime [1620-1807]” (= J. Mattoso, sous la dir., História de Portugal), Lisbonne, 1993.

7.L'influence des historiens du droit et de la pensée politique vient de plus loin, que l'on pense à la réception de P. MÊREA, de M. CAETANO ou de N. E. G. SILVA dans les groupes d'histoire des facultés des lettres, ou encore à Martim DE ALBUQUERQUE, dont la monographie (O Poder Político no Renascimento Português, Lisbonne, 1968) est vite entrée dans quelques programmes scolaires de l'histoire moderne du Portugal.

8. Nous nous référons aux influences des travaux de quelques-uns parmi les plus réputés de l'historiographie du politique en France : R. CAZELLES (1917-1985), R.-H. BAUTIER, B. GUENÉE, B. GUILLEMAIN, R. FÉDOU, J. FAVIER, P. CONTAMINE, F. AUTRAND, C. BEAUNE, H. MILLET, J.-P. GENET, C. GAUVARD, B. LEROY Ou A. RUCQUOI. On peut encore inclure les historiens du droit A. GOURON, A. RIGAUDIÈRE, G. GIORDANENGO et J. KRINEN ou l'historien spécialiste de la diplomatique O. GUYOTJEANNIN. Pour l'historiographie anglaise, on peut citer : P. S. LEWIS, M. G. VALE, C. ALLMAND, R. A. GRIFFITHS ou la réflexion méthodologique du moderniste W. REINHARD. Pour l'Italie, on peut mentionner les noms des historiens du droit comme M. ASCHERI, A. PADOA-SCHIOPPA ou M. SBRICOLI. Pour la Belgique, on peut citer les universitaires J. BARTIER, P. GODDING, J. PYCKE, H. SCHEPPER, J.-M. CAUCHIES ou E. BOUSMAR. Pour le pays voisin, en générations successives, on doit signaler J. A. MARAVALL (1908-1982), S. de MOXó (1921-1980), M. A. LADERO QUeSADA, J. M. NieTO SORIA et les histo-riens du droit J. M. PÉREZ-PRENDES, S. DE DIOS, D. TORRES SANZ, A. P. MARTíN, P. A. PorRAS ARBOlEDAS ou T. DE MONTAGUT. Enfin, pour l'historiographie nord-américaine voir, par exemple, J. R. STRAYER (1904-1987), F. J. Pegues, G. POST ou T. N. BISSON. Pour une vision de l'ensemble des principales références extérieures, Cf. A. L. de C. HOMEM, O Desembargo Régio (1320-1433), Porto, 1990, p. 599-618 et 621-629.

9.La notion de génération à laquelle nous nous reportons concerne des individus qui partagent le même "esprit du temps" pour utiliser les mots de J. ORTEGA Y GASSET (Cf. J. A. G. de FREITAS, “A Idade dos homens do poder : novos e velhos na burocracia de D. Afonso V (1439-1460)”, dans Antropológicas, 6, 2002, p. 173-179).

10.Cf. J.-P. GENET, “L'État Moderne : un modèle opératoire ?”, dans L'État Moderne : Genèse. Bilans et perspectives, Paris, 1990, p. 261-281; Id., "La genèse de l'État Moderne. Genèse d'un programme de recherche", dans M. H. da C. coElHo et A. L. de C. HOMEM, sous la dir., A Génese do Estado Moderno tardo-medievo. Ciclo de conferências, Lisbonne, 1999, p. 21-55. 11.Cf. A. de SouSA, “1325-1480”, dans A Monarquia feudal (= J. MATTOSo, sous la dir., História de Portugal, vol. 2), Lisbonne, 1994, p. 313-556. 
12.Particulièrement le cas des aires de frontière, où l'affirmation politique de la monarchie a une plus grande difficulté à s'implanter. Voir à ce sujet R. C. GOMES, "A Construção das fronteiras”, dans F. BETHENCOURT et D. R. CURTO, A Memória da Nação, Lisbonne, 1989, p. 357-382. Une étude bien plus développée peut être vue dans A. A. ANDRADE, Vilas, poder régio e fronteira : o exemplo do Entre Lima e Minho medieval, Lisbonne, 1994. Pour une approche générale, $C f$. EAD., "Estado, territórios e administração régia periférica", dans A Génese do Estado Moderno no Portugal tardo-medievo, op. cit., p. 151-187. 13.Sur l'administration municipale dans ses relations spécifiques avec le monarque, voir aussi M. H. da C. coelho et A. L. de C. HOMEM, sous la dir., Portugal em definição de fronteiras. Do condado Portucalense à crise do século XIV (= J. SERRÃo et A. H. de O. MARQUES, sous la dir., Nova História de Portugal, vol. 3), Lisbonne, 1996.

14.A. M. HESPANHA, "Centro e periferia nas estruturas administrativas do Antigo Regime”, Ler História, 8, 1986, p. 85-90. Récemment, l'auteur a attiré l'attention sur la question de l'efficacité de la machine administrative de la couronne affirmant, qu'au $\mathrm{XVI}^{\mathrm{e}}$ siècle, elle était "très faible" ( $C f$. Id., "O Estado Moderno na recente Historiografia portuguesa : historiadores do direito e historiadores "tout court", 1. A questão do Estado Moderno", dans A Génese do Estado Moderno no Portugal tardo-medievo, op. cit., p. 59 ss.).

15.B. LEROY, Sociedades e Poderes Políticos na Península Ibérica Medieval (séculos XIV-XV), Mem Martins, 2001.

16.J. MATTOSO, "Perspectivas actuais sobre a nobreza medieval portuguesa", Revista de História das Ideias, 19, 1997, p. 20. Dans ce dernier titre, il nous offre un bilan complet des études faites sur la noblesse dans les vingt dernières années.

17.Pour une approche générale des rapports entre l'Église et l'État, Cf. J. Matoso, "Igreja e Poder Régio”, dans A Génese do Estado Moderno no Portugal tardo-medievo, op. cit., p. 217-256.

18.R. C. GOMES procède à un premier bilan de la réapparition du politique dans "L'émergence du politique dans le Portugal du bas Moyen Âge", dans La recherche en histoire du Portugal, t. I, 1989, p. 24-32.

19.Ce travail dactylographié (mémoire de licence, 1974) est conservé à la Faculté des lettres de l'université de Porto ; Id., "Subsídios para o estudo da Administração Central no reinado de D. Pedro I", dans Id., Portugal nos finais da Idade Média, op. cit., p. 63-107. 20.0 Desembargo Régio (1320-1433), Porto, 1990.

21.Voir sa plus récente réflexion méthodologique : A. L. de C. HOMEM et J. A. G. DE FREITAS, “A Prosoprografia dos burocratas régios (séculos XIII-XV) : da elaboração à exposição dos dados", dans F. T. BARATA, Elites e Redes Clientelares na Idade Média. Problemas metodológicos. Actas do colóquio, Lisbonne/Évora, 2001, p. 171-210.

22.A. L. de C. HOMEM, "Conselho real ou conselheiros do rei ? A propósito dos "privados" de D. João I", Revista da Faculdade de Letras [UP]. História, II sér., IV, 1987, p. 9-68 (rééd. dans Id., Portugal nos finais da Idade Média, op. cit., p. 221-278).

23.Id., "Prólogo", dans Id., Portugal nos finais da Idade Média, op. cit., p. 7-12.

24.Titre d'un article qu'il a publié en France dans les années quatre-vingt : "L'État portugais et ses serviteurs (1320-1433)”, Journal des Savants, 1987, p. 181-203.

25.Outre ceux qui seront mentionnés ultérieurement, voir aussi M. MENDONÇA, D. João II. Um percurso humano e político nas origens da modernidade em Portugal, Lisbonne, 1995. Le projet d'une thèse de doctorat sur la bureaucratie manuéline, sous l'orientation de A. L. de C. HOMEM, se trouve à la FL/UP. 
26.J. A. G. DE FREITAS, A Burocracia do "Eloquente" (1433-1438). Os textos, as normas, as gentes, Cascais, 1996.

27.EAD., "Teemos por bem e mandamos". A burocracia Régia e os seus oficiais em meados de Quatrocentos (1439-1460), 2 vol., Cascais, 2001.

28.Voir les mémoires de maîtrise (FL/UP) présentés par : V. R. S. M. VAZ, A Boa memória do monarca. Os escrivães da Chancelaria de D. João I (1385-1433), 2 vol. polycopiés, Porto, 1995 ; A. P. P. G. ALMEIDA, A Chancelaria Régia e os seus oficiais em 1462, polycopié, Porto, 1996 ; A. P. C. BORLIDO, A Chancelaria Régia e os seus oficiais em 1463, polycopié, Porto, 1996 ; H. M. M. MONTEIRO, A Chancelaria Régia e os seus oficiais (1464-1465), 2 vol. polycopiés, Porto, 1997 ; A. E. T. de CARVALHO, A Chancelaria Régia e os seus oficiais em 1468, polycopié, Porto, 2001 ; H. A. R. CAPAS, A Chancelaria Régia e os seus oficiais no ano de 1469, polycopié, Porto, 2001 ; M. M. da S. DURÃo, 1471 : um ano africano no Desembargo de D. Afonso V, 2 vol. polycopiés, Porto, 2002 ; I. B. de C. HENRIQUES, Os Caminhos do Desembargo : 1472, um ano na burocracia do "Africano", 2 vol. polycopiés, Porto, 2001 ; E. G. D. FERREIRA, 1473 : um ano no Desembargo do Africano, 2 vol. polycopiés, Porto, 2001 ; et I. C. M. de BRITO, A Burocracia Régia tardo-Afonsina. A administração central e os seus oficiais em 1476, 2 vol. polycopiés, Porto, 2001. À cette liste, on peut ajouter les rapports inédits sur les greffiers et conseillers d'état des Chancelarias des rois Pedro I, Fernando, João et Afonso V. 29.Voir les chapitres sur les "Carreiras" et la "Memória da Idade" dans le premier volume du travail cité dans la note 27, p. 130-148 et 149-160.

30.J. A. G. DE FREITAS, “Teemos por bem e mandamos”, op. cit., t. I, p. 26.

31.Voir les réflexions méthodologiques dans : A. L. de C. HOMEM, "Dionisius et Alfonsus, Dei gratia reges et communis utilitatis gratia legiferi", Revista da Faculdade de Letras [UP]. História, II série XI, 1994, p. 11-110 ; Id., "Législation et compilation législative au Portugal du début du $\mathrm{XV}^{\mathrm{e}}$ siècle. La genèse des Ordonnances d'Alphonse V", dans F. AUTRAND, C. GAUVARD et J.-M. MOEGLIN, Saint-Denis et la Royauté. Études offertes à Bernard Guenée, Membre de l'Institut, Paris, 1999, p. 617-89 ; Id., "Estado Moderno e Legislação Régia : Produção e Compilação Legislativa em Portugal (XIII ${ }^{\mathrm{e}}-\mathrm{XV}^{\mathrm{e}}$ siècles)", dans $A$ Génese do estado Moderno no Portugal tardo-medievo, op. cit., p. 111-130 ; Id., "Ofício régio e serviço ao Rei em finais do século XV : norma legal e prática institucional”, Revista da Faculdade de Letras [UP]. História, II sér., XIV, 1997, p. 123-37 ; Id., "Poder e poderes no Portugal de finais da Idade Média", Praça Velha. Revista de Cultura da Cidade da Guarda, 3, 1998, p. 39-68 ; Id., "Este Reino a que o Gama voltou... : em torno da "modernidade" do Portugal manuelino", dans J. R. MAGALHÀEs et J. M. FLORES, Vasco da Gama : Homens, Viagens e Culturas. Actas do Congresso Internacional, t. I, Lisbonne, 2001, p. 495-512 ; Id., "Estado, instituições, poderes nos finais da Idade Média", Anais da UAL/série História, V-VI, 2000-2001, p. 349-355 ; Id., "Quando acaba a Idade Média ? A periodização da História institucional portuguesa dos séculos XV e XVI”, dans Turres Veteras ii. Actas de História Moderna, Torres Vedras, 2000, p. 9-19.

32.Justiça e Criminalidade no Portugal Medievo (1459-1481), Lisbonne, 1999. Cette publication ne comprend pas le deuxième volume de la version polycopiée de la thèse concernant les micro-biographies des officiers de justice ( $C f$. Justiça e criminalidade no Portugal Medievo (1450-1481), Thèse de doctorat dactylographiée, université de Porto, 1993).

33.Cf. A Guerra em Portugal nos finais da Idade Média, Lisbonne, 1998 ; Os Castelos portugueses nos finais da Idade Média. Presença, perfil, conservação, vigilância e comando, Lisbonne, 1999 et A Batalha de Aljubarrota, Lisbonne, 2003. 
34. Comme œuvre de référence, $C f$. P. CONTAMINE, sous la dir., Guerre et concurrence entre les États européens du XIVe au XVIII siècle, Paris, 1998.

35.Voir un regard pionnier sur les finances publiques médiévales dans A. H. DE MARQUES, “Fazenda Pública : na Idade Média”, dans J. SERRÃo, sous la dir., Dicionário de História de Portugal, t. II, Lisbonne, 1965, p. 189-191.

36.Pedidos e empréstimos públicos em Portugal durante a Idade Média, Lisbonne, 1964 ; EAD., "A Colheita régia medieval padrão alimentar de qualidade (Um contributo beirão)", dans Ias Jornadas de História Regional do distrito de Castelo Branco, Castelo Branco, 1987, p. 175-189 ; EAD., “Alguns aspectos da visita régia ao Entre Cávado e Minho, no século XIII", Estudos Medievais, 10, 1993, p. 33-57.

37.Cf. A Génese do Estado Moderno no Portugal tardo-medievo, op. cit., p. 95-110.

38.As Cortes Medievais Portuguesas (1385-1490), 2 vol., Porto, 1990 ; Id., "O Parlamento Medieval Português : perspectivas novas", Revista da Faculdade de Letras [UP], História, II série, VII, 1990, p. 47-58 ; Id., "Estado e Comunidade : Representação e Resistências", dans A Génese do Estado Moderno no Portugal tardo-medievo, op. cit., p. 293-313 ; Id., "O Discurso político dos concelhos nas Cortes de 1385", Revista da Faculdade de Letras [UP]. História, II série, II, Porto, 1985.

39.Voir B. GUENÉE, "Les tendances actuelles de l'histoire politique du Moyen Âge français", dans Tendances, perspectives et méthodes de l'histoire médiévale, Actes du $100^{e}$ Congrès national des sociétés savantes, Paris, 1977, p. 45-70.

40.Thèse de doctorat présentée en 1993 (UNL), publiée à Lisbonne en 1995 sous le même titre donné. Traduction anglaise : The Making of a Court Society. Kings and Nobles in Late Medieval Portugal, Cambridge, 2003.

41. Rappelons que l'histoire des rituels est considérée comme un objet même de l'histoire médiévale en Allemagne - domaine où l'école allemande est pionnière - et s'étend à l'histoire des représentations ( $C f$. C. GAUVARD, "Le rituel, objet d'histoire" dans J.-C. SCHMitT et O. G. OEXLE, sous la dir., Les tendances actuelles de l'histoire du Moyen Âge en France et en Allemagne, Paris, 2003, p. 269-281).

42.R. C. GOMES, "Monarquia e território : residências reais portuguesas, séculos XIV a XVI", dans G. SABATIER et alii, Lugares do poder. Europa séculos XV a XX. Lieux de pouvoir. Europe $X V^{e}-X X^{e}$. Places of power. Europe 15th-20th centuries, Lisbonne, 1998, p. 84-105 ; J. C. V. DA SILVA, "Palácio de Sintra : o poder de um lugar, séculos XV a XIX”, dans ibid., p. 204-225. Dans une même perspective, voir également J. A. G. DE FREITAS, "O Portugal Atlântico e o Portugal Mediterrânico na itinerância régia por meados de Quatrocentos", à paraître dans le volume d'hommage au professeur J. Marques, Porto [sous presse]. 43.Un bilan des développements récents de cette matière se trouve dans M. H. da C. COELHo, “A Diplomática em Portugal : balanço e estado actual”, Revista Portuguesa de História, 26, 1991, p. 122-155.

44.Voir les références bibliographiques présentes dans A. L. de C. HOMEM, “O Medievismo em liberdade", op. cit.

45.M. H. da C. COELHO, "A História Medieval Portuguesa : caminhos percorridos e a percorrer”, Media Actas. Boletim do Núcleo de História Medieval, Ponta Delgada, 1990, p. 1-17 ; Id. "O Estado e as Sociedades Urbanas", dans A Génese do Estado Moderno no Portugal tardo-medievo, op. cit., p. 269-292.

46.Une approche bibliographique sur le sujet se trouve dans H. B. MORENO, L. M. DUARTE et L. C. do AMARAL, "História da Administração Portuguesa na Idade Média : um balanço", Ler História, 21, 1991, p. 24-34. 
47.Voir une synthèse dans M. H. da C. coelHo, "Les élites municipales", Anais de l'Université Autónoma de Lisbonne/série História, II, 1995, p. 23-27.

48. Identificação de um país. Ensaio sobre as origens de Portugal. 1065-1325, Lisbonne, 1985, t. I (Oposição), p. 289 ss. et t. II (Composição), p. 166-176.

49.0 Poder concelhio : das origens às Cortes Constituintes. Notas da História Social, Coïmbre, 1986.

50.En deux volumes autonomes. Pour le moment, c'est surtout le premier volume qui nous intéresse, dont le titre est Notas do viver social, Lisbonne, 1990. Voir aussi "O Estado e as Sociedades Urbanas", dans A Génese do Estado Moderno no Portugal tardo-medievo, op. cit., p. 269-292.

51.Voir les références bibliographiques dans A. L. de C. Homem, “O Medievismo em liberdade", op. cit.

52.Voir, de ce dernier auteur, la publication conjointe de onze études, de 1978 à 1996, dans le volume Um olhar sobre a cidade medieval, Cascais, 1996. Malgré ce fait, une référence spéciale pour les travaux pionniers de J. Marques, “A Administração municipal de Vila do Conde em 1466", Bracara Augusta, XXXVII [83-84], 1983, p. 5-115 et “A Administação municipal de Mós de Moncorvo en 1439”, Brigantia, 5, 1985, p. 515-560. 53. "Vereação" e "Vereadores" : o governo do Porto em finais do século XV, Porto, 1993 ; Id., Projecção espacial de domínios : das relações de poder ao burgo portuense (1385-1502), Thèse de doctorat dactylographiée, Lisbonne, 1999.

54.Organização dos Poderes e Estrutura Social. A ilha da Madeira : 1460-1521, Cascais, 1996. 55.Voir les références bibliographiques dans A. L. de C. HOMEM, “O Medievismo em liberdade", op. cit.

56.Sur ce sujet, voir J. MARQUES, M. H. da C. COELHO et A. L. de C. HOMEM, "Diplomatique municipale portugaise (XIII ${ }^{\mathrm{e}}-\mathrm{XV}^{\mathrm{e}}$ siècles)", dans W. PREVENIER et Th. De HEMPTINNE, La diplomatique urbaine en Europe, Louvain/Apeldoorn, 2000, p. 281-305.

57. A Nobreza Medieval Portuguesa. A familia e o poder, Lisbonne, 1987, 2 ; Id., Ricos-homens, Infações e Cavaleiros. A nobreza medieval portuguesa nos séculos XI a XII, Lisbonne, 1985 ; Id., “A Nobreza e a Revolução de 1383", dans Id., Fragmentos de um composição medieval, Lisbonne, 1988, p. 115-123. Pour une information plus détaillée, voir la bibliographie finale dans Id., "Perspectivas actuais sobre a nobreza medieval portuguesa", Revista de História das Ideias, 19, 1997, p. 29-37.

58. A Concepção nobiliárquica do espaço ibérico (1280-1380), Lisbonne, 1994.

59.Depois de espectáculo trovadoresco. A estrutura dos cancioneiros peninsulares e as recolhas dos séculos XIII e XIV, Lisbonne, 1994 (Thèse de doctorat présentée à l'Université de Coïmbre en 1992).

60.L. KRUS, "O Discurso sobre o passado na legitimação do senhorialismo português dos finais do século XIII”, dans Id., Passado, Memória e Poder na Sociedade Medieval Portuguesa. Estudos, Redondo, 1994, p. 197-207 ; A R. de OLIVEIRA, Trobadores e xograres. Contexto histórico, Vigo, 1995.

61.A Nobreza de Corte no reinado de D. Afonso III, Thèse de doctorat dactylographiée, 2 vol., univeristé de Coïmbre, 1992.

62.0s Pimentéis. Percursos de uma linhagem medieval portuguesa (Séculos XIII-XIV), Lisbonne, 2000.

63.Os Patronos do mosteiro de Grijó : evolução e estrutura da familia nobre medieval, séculos XIXIV, Ponte de Lima ; Id., Linhagens medievais portuguesas. Genealogias e Estratégias (1279-1325), 3 vol., Porto, 1999. 
64.Le projet est sous la responsabilité de A. H. de O. MARQUES, dans le cadre du Centre d'études historiques de l'université Nova de Lisbonne.

65. A Casa senhorial do Infante D. Henrique, Lisbonne, 1991.

66. Linhagem, parentesco e poder : a casa de Bragança (1384-1483), Lisbonne, 1990.

67. A Casa dos Coutinhos : Linhagens, Espaço e Poder (1360-1452), Cascais, 1999.

68.0 Morgadio em Portugal, sécs. XIV-XV. Modelos e práticas de comportamento linhagístico, Lisbonne, 1995.

69.Il s'agit, en particulier, de J. Le GOFF, J.-C. SCHMITT ou A. BOUREAU.

70.A Arquidiocese de Braga no seculo XV, Lisbonne en 1988.

71. “Igreja e Poder Régio", dans A Génese do Estado Moderno no Portugal tardo-medievo, op. cit., p. 217-255.

72.Igreja e poder régio no século XV. Dinastia de Avis e liberdades eclesiásticas (1383-1450), Lisbonne, 1997 (travail présenté à la Faculté des lettres de Lisbonne en 1993) ; EAD., Estudos sobre o poder (séculos XIV-XVI), Lisbonne, 2003.

73.As Dimensões de um poder. A diocese de Évora na Idade Média, Lisbonne, 2003.

74.- Cf. les références bibliographiques dans A. L. de C. HOMEM, "O Medievismo em liberdade", op. cit.

75.L. KUCHENBUCH, cité dans P. MONNET, “Conclusions”, dans Les tendances actuelles..., op. cit., p. 631.

76.En 1988, A. L. de C. HOMEM, A. A. ANDRADE et L. C. AMARAL, avaient prévu que l'histoire des pouvoirs serait une ligne d'investigation prometteuse, ce qui est confirmé aujourd'hui. Voir leur étude conjointe "Por onde vem o medievismo em Portugal ?", Revista de História Económica e Social, 22, 1988, p. 115-138.

77.F. cAtroga, "Fim da História ou das Filosofias da História ?", dans Id., Caminhos do Fim da História, Lisbonne, 2003, p. 141-161.

78.J. мAтTOSo, “A História hoje : que história ensinar ?”, dans Um século de Ensino da História..., op. cit., p. 221-236.

79.De préférence des thèmes tels que la mort, la sexualité, le corps ou la spiritualité. Cf., parmi d'autres, A. L. de C. Hомем, “O Medievismo em liberdade (anos 70-anos 90)", dans Um século de Ensino da História..., op. cit. p. 211 ss. L'auteur attire l'attention sur les domaines de pointe, les situant dans le cadre de l'histoire des mentalités. y compris des mentalités politiques. et sous l'influence de l'anthropologie.

80.Sur les récents domaines de la recherche en France et en Allemagne et leurs influences respectives, voir Les tendances actuelles de l'histoire de Moyen Âge, op. cit.

INDEX

Mots-clés : pouvoir

Index géographique : Portugal 